\title{
Super-LOTIS/LOTIS/LITE: Prompt GRB Followup Experiments
}

H.S. Park, E. Ables, S. Barthelmy, M. Bradshaw, T. Cline, N. Gehrels, D. Hartmann, K. Hurley, R. Nemiroff, W. Pereira, D. Perez-Ramirez, G.G. Williams, K. Ziock

This article was submitted to Gamma 2001 Symposium, Baltimore, MD, April 4-6, 2001

\section{June 25, 2001}

Lawrence

Livermore

National

Laboratory 


\section{DISCLAIMER}

This document was prepared as an account of work sponsored by an agency of the United States Government. Neither the United States Government nor the University of California nor any of their employees, makes any warranty, express or implied, or assumes any legal liability or responsibility for the accuracy, completeness, or usefulness of any information, apparatus, product, or process disclosed, or represents that its use would not infringe privately owned rights. Reference herein to any specific commercial product, process, or service by trade name, trademark, manufacturer, or otherwise, does not necessarily constitute or imply its endorsement, recommendation, or favoring by the United States Government or the University of California. The views and opinions of authors expressed herein do not necessarily state or reflect those of the United States Government or the University of California, and shall not be used for advertising or product endorsement purposes.

This is a preprint of a paper intended for publication in a journal or proceedings. Since changes may be made before publication, this preprint is made available with the understanding that it will not be cited or reproduced without the permission of the author.

This report has been reproduced directly from the best available copy.

Available electronically at http://www.doe.gov/bridge

Available for a processing fee to U.S. Department of Energy and its contractors in paper from

U.S. Department of Energy

Office of Scientific and Technical Information

. P.O. Box 62

Oak Ridge, TN 37831-0062

Telephone: (865) 576-8401

Facsimile: (865) 576-5728

E-mail: reports@adonis.osti.gov

Available for the sale to the public from

U.S. Department of Commerce

National Technical Information Service

5285 Port Royal Road

Springfield, VA 22161

Telephone: (800) 553-6847

Facsimile: (703) 605-6900

E-mail: orders@ntis.fedworld.gov

Online ordering: http://www.ntis.gov/ordering.htm

\section{OR}

Lawrence Livermore National Laboratory

Technical Information Department's Digital Library

http://www.llnl.gov/tid/Library.html 


\title{
Super-LOTIS / LOTIS / LITE: Prompt GRB Followup Experiments
}

\author{
H. S. Park ${ }^{1}$, E. Ables', S. Barthelmy ${ }^{2}$, M. Bradshaw ${ }^{3}$, T. Cline ${ }^{2}$, N.

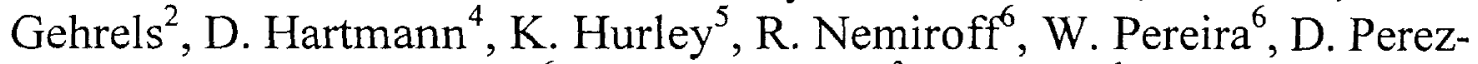 \\ Ramirez $^{6}$, G. G. Williams ${ }^{3}$, K. Ziock ${ }^{1}$ \\ 'Lawrence Livermore National Laboratory, Livermore, CA 94550 \\ ${ }^{2}$ NASA/Goddard Space Flight Center, Greenbelt, MD 20771 \\ ${ }^{3}$ Steward Observatory, Tucson, AZ 85721 \\ ${ }^{+}$Dept. Of Physics and Astronomy, Clemson University, Clemson, SC 29634 \\ ${ }^{5}$ Space Science Laboratory, University of California, Berkeley, CA 94720 \\ ${ }^{\circ}$ Dept. Of Physics, Michigan Technological University, Houghton, MI 49931
}

\begin{abstract}
LOTIS (Livermore Optical Transient Imaging System) and Super-LOTIS are automatic telescope systems that measure very prompt optical emission occurring within seconds of the gamma-ray energy release during a Gamma Ray Burst (GRB). Unlike hour-todays delayed afterglow measurements, very early measurements will contain information about the GRB progenitor. To accomplish this, we developed and have been operating automated telescopes that rapidly image GRB coordinate error boxes in response to triggers distributed by the GRB Coordinate Distribution Network (GCN). LOTIS, located in California, consists of 4 cameras each with a different astronomical filter (B, V, R, open) that can respond to GRB triggers within $5 \mathrm{~s}$. Super-LOTIS can point to any part of the sky within $30 \mathrm{~s}$ upon receipt of a GCN trigger and its sensitivity is as deep as $V=17 \sim 19$ depending on the integration times. Since the shutdown of the CGRO, there has been no real-time GRB triggers that enable the LOTIS systems to measure real-time GRB counterpart fluxes as of May 2001. This paper describes performance of these systems. We also present our plan to replace the current optical CCD camera on the Super-LOTIS to a near infrared camera to be able to probe dusty GRB environment.
\end{abstract}

\section{INTRODUCTION}

The dramatic breakthrough in our understanding of GRBs occurred when the high resolution X-ray detector on the Beppo/SAX satellite was able to determine the position of a GRB with sufficient accuracy to enable a large telescope to observe a faint, fading afterglow days later. Optical and radio afterglows now have been observed for many GRBs during the last two years. These long-lasting but faint afterglows have been successfully explained in the "fireball models" as the result of the synchrotron interaction with surrounding material'.

However, prompt counterparts associated with GRBs are rare. Rapidly slewing and automatic telescopes such as LOTIS $^{2}$ and ROTSE ${ }^{3}$ have been operating to catch early glimpse of the GRBs detected by the BATSE instrument. After running these telescopes for many years recording real-time images of the GRB error boxes, only 
one event (GRB990123) has been detected simultaneously with a GRB. But this event was unusual in terms of its total X-ray fluence, peak flux and spectrum ${ }^{4}$. Unlike the observed later-time afterglows, prompt optical measurements (or even stringent constraints on that optical emission) would provide information about the GRB progenitors. In order to detect many more prompt counterparts, we are currently operating two instruments, LOTIS and Super-LOTIS, connected to the Coordinate Distribution by the current and future satellites such as the HETE-2, Rossi-XTE, INTEGRAL, and Swift missions.

\section{SUPER-LOTIS}

The Super-LOTIS telescope is a Boller \& Chivens $0.6 \mathrm{~m} \mathrm{f} / 3.5$ reflector telescope. It has superb optical quality and is equipped with computer controllable drives. Its focal array is a Loral $2048 \times 2048$ pixel $15 \times 15 \mu \mathrm{m} /$ pixel $\mathrm{CCD}$ cooled to $-30^{\circ} \mathrm{C}$ with custom-built readout electronics. This focal plane array is placed at the primary focus of the mirror with a coma corrector yielding $0.84 \times 0.84^{\circ}$ field of view. Super-LOTIS began operation in October 2000 at Kitt Peak National Observatory in Arizona. Figure 1 shows its operation inside a roll-off roof housing.

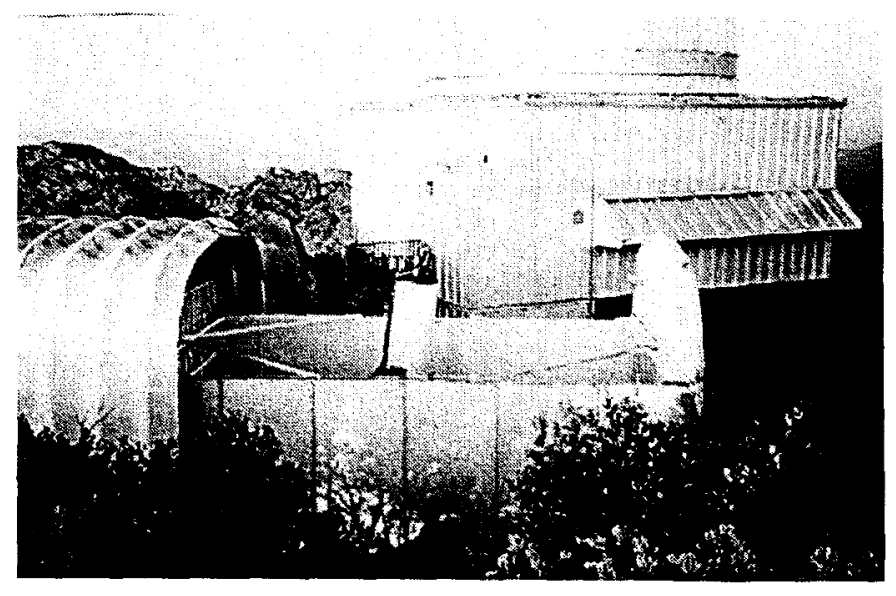

FIGURE 1. Super-LOTIS in operation. It is housed in a building with a roll-off roof and is fully automated.

Since there were no real-time GRB triggers available until the end of May, 2001, Super-LOTIS has been responding to Beppo/SAX and IPN GRB triggers that usually come many hours after the burst. After this delay, the relativistic shock wave from the GRB has interacted with the interstellar medium producing an afterglow that decays as a power-low. Super-LOTIS imaged one of these afterglows, GRB010222, on Feb. 23, 2001. The image was taken 23.6 hours after the burst and the brightness of the afterglow was $R=20.0$. The Super-LOTIS image of this afterglow is shown in Figure 2. When it is not imaging the GRB fields, it systematically searches for many other classes of transient objects, such as novae and supernovae. It will also monitor longand short-period stellar variability. 


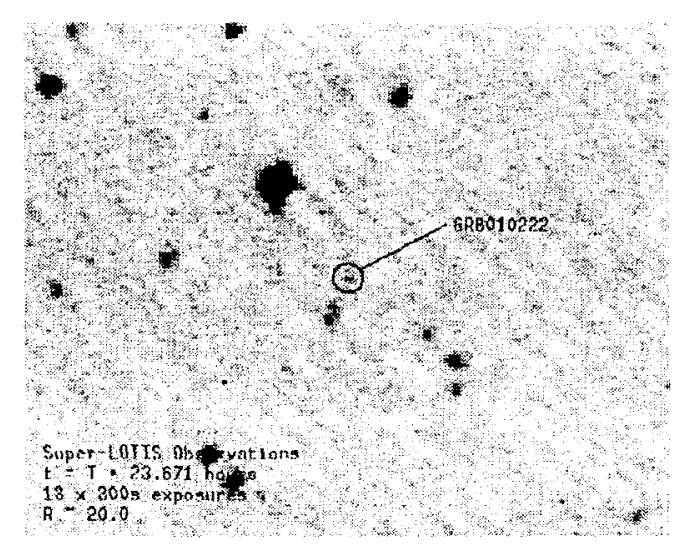

FIGURE 2. Super-LOTIS response to GRB010222.

\section{LOTIS}

The LOTIS was originally constructed to respond rapidly to real-time GRB triggers provided by the BATSE which had a $1-\sigma$ error of $2 \sim 10^{\circ}$. It utilizes commercially available Canon $\mathrm{f} / 1.8$ with a $200 \mathrm{~mm}$ focal length lenses. With a $2048 \times 2048$ pixel 15 $\mathrm{x} 15 \mu \mathrm{m} /$ pixel CCD array, one camera images $8.8 \times 8.8^{\circ}$ field-of-view. The mount can point any part of the sky within $5 \mathrm{~s}$ after it receives a trigger. We have a $2 \times 2$ array of such cameras originally configured to have $17.4 \times 17.4^{\circ}$ field-of-view for BATSE triggers. Results from LOTIS have been published ${ }^{5}$. Recently we collapsed the angular offsets of this system to have all 4 cameras view the same patch of the sky but each with a different astronomical filter, R, V, B or clear filter, for simultaneous color imaging of a GRB counterpart. Its sensitivity is mag 13 to 15 depending on the integration times, weather and filter types. LOTIS is located at a LLNL's test facility, 25 miles east of Livermore, California. The system is fully automated to respond the HETE-2 triggers and is operating every night when the weather permits.

\section{LITE}

The LITE (Livermore Infrared Transient Experiment) is our planned upgrade system for the Super-LOTIS. Measured GRB distance scales, recent conjecture of their star-forming environment and theoretical arguments of their high red-shifts ${ }^{6}$ suggest that the optical signal may be obscured compared to the infrared signals. In addition, all the future planned GRB missions lack coverage of the infrared signals from a GRB. In order to fill this gap, we plan to upgrade the Super-LOTIS by replacing the current optical CCD camera with an infrared camera. We will install a $\mathrm{f} / 2$ secondary mirror in place of the current coma corrector and a HgCdTe $512 \times 512$ 
pixel camera for the focal plane array with a filter wheel equipped with $\mathrm{J}, \mathrm{H}$ and $\mathrm{K}$ filters. This system will have $6.7 \times 6.7$ arcmin field-of-view well-matched to the Swift error box of 4 arcmin. Its expected sensitivity and prompt response capability will be the same as the Super-LOTIS system.

\section{SUMMARY}

Table 1 summarizes the parameters for the automatic telescopes discussed in this paper. Prompt optical and infrared counterparts of the GRB will provide important clues to understanding of the GRB progenitors. With our current operating LOTIS and Super-LOTIS and planned LITE systems connected to the real-time GRB triggers from the space-borne GRB detectors, we will measure many early-time optical and infrared fluxes associated with the GRBs.

TABLE 1. LOTIS System Parameters.

\begin{tabular}{|c|c|c|c|}
\hline Instrument & Super-LOTIS & LOTIS & LITE \\
\hline Aperture Diameter & $0.6 \mathrm{~m}$ & $0.11 \mathrm{~m}$ & $0.6 \mathrm{~m}$ \\
\hline Optics Speed & $\mathrm{f} / 3.5$ & $\mathrm{f} / 1.8$ & $\mathrm{f} / \mathbf{8}$ \\
\hline Imaging Sensor & $2048 \times 2048 C C D$ & 4 of $2048 \times 2048 \mathrm{CCD}$ & $512 \times 512 \mathrm{HgCdTe}$ \\
\hline Field-of-View & $0.84 \times 0.84^{\circ}$ & $8.8 \times 8.8^{\circ}$ & $6.7 \times 6.7$ arcmin \\
\hline Resolution & 1.5 arcsec/pixel & 15 arcsec/pixel & 0.78 arcsec/pixel \\
\hline Sensitivity & $R \sim 17$ (5 s integration) & $V \sim 14(10 \mathrm{~s}$ integration) & $\mathrm{J} \sim 17$ \\
\hline Filters & $\mathbf{B}, \mathbf{V}, \mathbf{R}$, clear & B, V, R, clear & $\mathbf{J}, \mathbf{H}, \mathbf{K}$ \\
\hline Response time & $<\mathbf{3 0 s}$ & $<5 \mathrm{~s}$ & $<\mathbf{3 0 s}$ \\
\hline
\end{tabular}

\section{ACKNOWLEDGMENTS}

This research is supported under NASA contract numbers S-03975G and S-57797F and under the auspices of the U.S. Department of Energy by University of California Lawrence Livermore National Laboratory under contract No. W-7405-Eng-48.

\section{REFERENCES}

1. Wijers, R. A. M J., et al., 1997, MNRAS 288, L51.

2. Park, H., et al., Explosive Phenomena in Astrophysical Compact Objects, edited by H. Chang et al., AIP Conference Proceedings 556, New York: American Institute of Physics, 2000, p. 261.

3. Akerlof, C., et al., ApJ, 2000, 532, L25.

4. Briggs, M., et al., ApJ, 2000, 524, 82.

5. Williams, G., et al., Gamma-Ray Bursts, edited by M. Kippen et al., AIP Conference Proceedings 526, New York: American Institute of Physics, 2000, p. 250.

6. Lamb, D., et al., ApJ, 2000, 536, 1. 